\title{
Changes in norovirus genotype diversity in gastroenteritis outbreaks in Alberta, Canada: 2012-2018
}

\author{
Maria E. Hasing ${ }^{1}$, Bonita E. Lee², Yuanyuan Qiu' ${ }^{1}$ Ming Xia ${ }^{3}$, Kanti Pabbaraju ${ }^{5}$, Anita Wong ${ }^{5}$, Graham Tipples ${ }^{4,5}$, \\ Xi Jiang ${ }^{3}$ and Xiaoli L. Pang ${ }^{1,5^{*}}$
}

\begin{abstract}
Background: The emergence of norovirus genotype Gll.4 variants has been associated with gastroenteritis pandemics worldwide, prompting molecular surveillance for early detection of novel strains. In this study, we aimed to analyze the outbreak activity of norovirus and characterize the norovirus strains circulating in Alberta between July 2012 and February 2018.

Methods: Stool samples from gastroenteritis outbreaks in Alberta were tested for norovirus at the Provincial Laboratory for Public Health using a multiplex real time-RT PCR assay. The ORF1 and ORF2-genotypes of norovirus positive samples were assigned based on phylogenetic analyses of partial polymerase and capsid sequences, respectively.

Results: A total of 530 norovirus outbreaks were identified. During July 2012 and June 2017 there was a gradual decrease in the annual number of Gll.4 outbreaks, however, outbreak numbers increased from June 2017-February 2018. Four novel strains emerged: Gll.17 Kawasaki in July 2014-June 2015, GII.P16/GIl.4 Sydney in July 2015-June 2016, GII.P16/GII.2 and GII.P4 New Orleans/GII.4 Sydney in July 2016-June 2017. GII.Pe/GIl.4 Sydney was the single predominant strain responsible for the majority (over 50\%) of all norovirus outbreaks up to June 2015. Between June 2017 and February 2018, GII.P16/GII.4 Sydney was the leading strain causing 63\% of all norovirus outbreaks.

Conclusions: Gll.4 stands as the predominant capsid genotype causing a large majority of the norovirus outbreaks in early 2018. An increase in genotype diversity was observed in the last years, characterized by a high circulation of non-Gll.4 strains and Gll.4 recombinants.
\end{abstract}

Keywords: Norovirus, Alberta, Canada, Gastroenteritis, Outbreaks, Humans, Molecular epidemiology

\section{Background}

With advancement in molecular testing, Norovirus (NoV) is recognized as the leading cause of morbidity and mortality from diarrhoeal disease across all ages [1]. Approximately $18 \%$ of all acute gastroenteritis around the world is caused by $\mathrm{NoV}$ with an estimated economic burden of 60 billion US dollars per year [2, 3]. Norovirus has a single-stranded positive-sense RNA genome of $\sim 7.5 \mathrm{~Kb}$ encompassing three open reading frames (ORFs). The three ORFs encode a non-structural polyprotein, the

\footnotetext{
*Correspondence: Xiao-Li.Pang@albertapubliclabs.ca

'Department of Laboratory Medicine and Pathology, University of Alberta, Edmonton, AB, Canada

${ }^{5}$ Provincial Laboratory for Public Health (Microbiology), University of Alberta Hospital, WMC 2B4.58, 8440-112 Street, Edmonton, Alberta T6G 2J2, Canada Full list of author information is available at the end of the article
}

major capsid gene (VP1) and the minor capsid gene (VP2).

The NoV genome has high genetic variability at the P2 subdomain of VP1. P2 forms the outermost part of the virion and contains binding sites for histo-blood group antigens (HBGAs), attachment factors for human $\mathrm{NoV}$ strains. Norovirus can evade host immune responses by antigenic drift, through a process similar to influenza's epochal evolution [4]. Recombination is another mechanism of evolution for NoV that often occurs at the ORF1/ ORF2 junction, further increasing the genetic diversity of the virus [5]. Seven NoV genogroups (GI to GVII) have been described to date of which GI, GII and GIV can cause gastroenteritis in humans, with GIV being less common [6]. NoV genogroups are sub classified into

(c) The Author(s). 2019 Open Access This article is distributed under the terms of the Creative Commons Attribution 4.0 International License (http://creativecommons.org/licenses/by/4.0/), which permits unrestricted use, distribution, and reproduction in any medium, provided you give appropriate credit to the original author(s) and the source, provide a link to the Creative Commons license, and indicate if changes were made. The Creative Commons Public Domain Dedication waiver (http://creativecommons.org/publicdomain/zero/1.0/) applies to the data made available in this article, unless otherwise stated. 
genotypes; at least 14 ORF1-based and 9 ORF2-based genotypes have been described for GI and 27 ORF1-based and 22 ORF2-based genotypes for GII [6].

NoV GII.4 has been the most common genotype in circulation worldwide since the mid-1990s. New genetic clusters or variants of NoV GII.4 have emerged periodically and caused pandemics including: US95/96 in 1996, Farmington Hills in 2002, Hunter in 2004, Den Haag in 2006, New Orleans in 2010 and Sydney in 2012 [7-9]. No global pandemic GII.4 strain has yet emerged after GII.4 Sydney. A novel cluster of GII.17 named Kawasaki became predominant in Asia during the 2014-2015 winter season [10] raising concern over a possible new global pandemic NoV genotype. Since its emergence in Asia, GII.17 Kawasaki has also been reported in other continents although with lower prevalence [10].

It is yet unknown why GII.4 has remained as the predominant genotype for over two decades but several features of this genotype support its enhanced circulation, including higher rates of evolution and a progressive accumulation of mutations that help evade host immune responses [11, 12]. Moreover, pandemic GII.4 variants can broadly bind to a wide set of HBGAs, a feature that favours virus transmission by providing $\mathrm{NoV}$ a large pool of individuals genetically susceptible to infection [13].

The aim of the present study is to describe the outbreak activity of NoV from July 2012 to February 2018 in Alberta, Canada. We provide information on the norovirus genotypes in circulation and their relevance in outbreak settings. This study provides important data for vaccine development and enhances our understanding of norovirus disease burden.

\section{Methods}

\section{Samples}

Gastroenteritis outbreak investigations in Alberta were managed by public health officials in collaboration with the Provincial Laboratory for Public Health (ProvLab) [8]. Stool samples collected between July 2012 and February 2018 during outbreak investigations were tested at ProvLab for NoV genogroup I and genogroup II using a real time RT-PCR assay [14]. A NoV- confirmed outbreak was defined as $\geq 2$ epidemiologically linked cases with gastroenteritis and at least one sample tested positive for NoV. Data was analyzed from July to June of the following year considering the winter seasonality of NoV.

Outbreak settings were classified into 6 different groups: 1) community long-term care, hospital longterm care, supportive living, and group homes facilities; 2) hospital acute care; 3) food establishments, catering events, food shops, community functions, conferences and hotels; 4) day care centers; 5) other types of group residences, e.g. camp, dormitory; and 6) other settings including community shelters, community services, household, schools and cruise ships. Groups 1 and 2 were classified as healthcare-related settings, whereas groups 3 to 6 were classified as non-healthcare-related.

\section{Norovirus genotyping}

For each NoV-positive outbreak, one NoV positive sample was selected for genotyping. Briefly, the nucleic acid extract from each stool sample was subjected to reverse transcription (RT) with random primers and the resulting cDNA was PCR-amplified. Samples collected up to February 2017, were amplified in region C (ORF2) using primer pair G2SKF/G2SKR for genogroup II strains or primer pair G1SKF/G1SKR for genogroup I strains [15]. Samples with emergent or unassigned genotypes based on region $\mathrm{C}$ sequence analysis were further genotyped targeting the 3'end of the polymerase gene using primers LV4282-99F [16] and COG2R [17]. Samples collected from March 2017 onwards, were genotyped using a dual polymerase-capsid genotyping protocol based on a single PCR amplicon obtained with primer pair MON432/ G1SKR for genogroup I strains and primer pair MON431/G2SKR for genogroup II strains [18]. All PCR products were subjected to Sanger sequencing and genotypes were assigned using the Norovirus Genotyping tool [19]. A large majority of strains from outbreaks occurring between July 2012 and June 2015 were left uncharacterized at ORF1; retrospective characterization was not attempted based on observations that $69 \%$ of norovirus GII outbreaks in Alberta were caused by a single ORF2 genotype, GII.4 Sydney, and reports from North America [18] and diverse countries from different continents [20] suggest that GII.Pe/GII.4 Sydney was the major strain in circulation worldwide during that time frame.

\section{Phylogenetic analysis}

Phylogenetic analyses of GII.17, GII.P16/GII.4 Sydney, GII.P4 New Orleans/GII.4 Sydney and GII.P16/GII.2 sequences obtained in our study were performed with MEGA 6.06 [21]. Maximum likelihood trees were constructed using the substitution model producing the lowest Bayesian Information Criterion scores, as calculated by the software. For all trees, branch significance was estimated based on 1000 bootstrap replicates.

\section{Cloning, expression and purification of recombinant $P$ domain proteins}

The capsid P domain of two GII.P16/GII.4 Sydney outbreak strains (AB-2016-26 and AB-2016-190) were amplified by RT-PCR using forward primer ACGCGGATC CTCAAGAACTAAACCATTCTCTGTCC and reverse primer ATAAGAATGCGGCCGCTTAGCAAAAGCAA TCGCCACGGCAATCGCATACTGCACGTCTACGCC CCGTTCC and cloned into pGEX-4 T-1 vector (GST 
Gene fusion System, GE Healthcare Life Sciences) between the Bam $\mathrm{HI}$ and Not I sites. An RGD4C tag (CDCRGDCFC) was linked to the $\mathrm{C}$ terminus of the $\mathrm{P}$ domain for $\mathrm{P}$ particle formation [22]. The recombinant P domain protein was expressed in E. coli (BL21, DE3) with induction by $0.25 \mathrm{mM}$ isopropyl- $\beta$ - $D$-thiogalactopyranoside (IPTG) at room temperature $\left(\sim 21^{\circ} \mathrm{C}\right)$ overnight as described elsewhere [22]. Purification of the glutathione S-transferase (GST)-P domain fusion protein was performed using resin of Glutathione Sepharose 4 Fast Flow (GE Healthcare Life Sciences) according to the manufacturer's instruction. GST was removed from the target proteins by thrombin (GE Healthcare Life Sciences) cleavage either on beads or in solution (phosphate buffer saline, PBS, $\mathrm{pH}$ 7.4).

\section{Saliva binding assay of P-domain proteins}

The saliva-based binding assays were performed as previously described [13]. Briefly, boiled human saliva with known HBGA phenotypes collected from Cincinnati, $\mathrm{OH}$, United States, were diluted 1000-fold and used to coat 96-well microtiter plates (Dynex Immulon; Dynatech, Franklin, MA). After blocking with 5\% non-fat milk in PBS, different concentrations of P-domain protein $(15,7.5,3.75 \mathrm{ng} / \mu \mathrm{l})$ were added to the wells. The bound $\mathrm{P}$ proteins were detected using a guinea pig anti-NoV antiserum (1:3000), followed by the addition of HRP-conjugated goat anti-guinea pig IgG. The HRP activity was then measured with TMB kit (Kierkegaard \& Perry Laboratories, Gaithersburg, MD) and the OD450 values were read with an ELISA spectrum reader (Tecan, Durham, NC).

\section{Statistical analysis}

The proportion of NoV GI and GII outbreaks by settings were compared using the Chi-square exact test. The annual numbers of NoV positive outbreaks occurring between July 2012 and June 2017 were compared to those occurring in the previous 5 years, from July 2007 to June 2012, using a one tailed t-test, and a significance of $p<0.05$.

\section{Results}

\section{Norovirus outbreaks: Annual activity and circulating} genotypes

A total of 1572 gastroenteritis outbreak investigations were performed in Alberta between July 1st 2012 and February 30th 2018, of which 859 (54.6\%) had specimens submitted to the ProvLab for laboratory testing. Norovirus was identified in $530(61.7 \%)$ of all tested outbreaks. The monthly distribution of NoV-positive outbreaks peaked in the winter months (Fig. 1), however, spring peaks with higher activity than that of winter months occurred in March 2014 and May 2016.

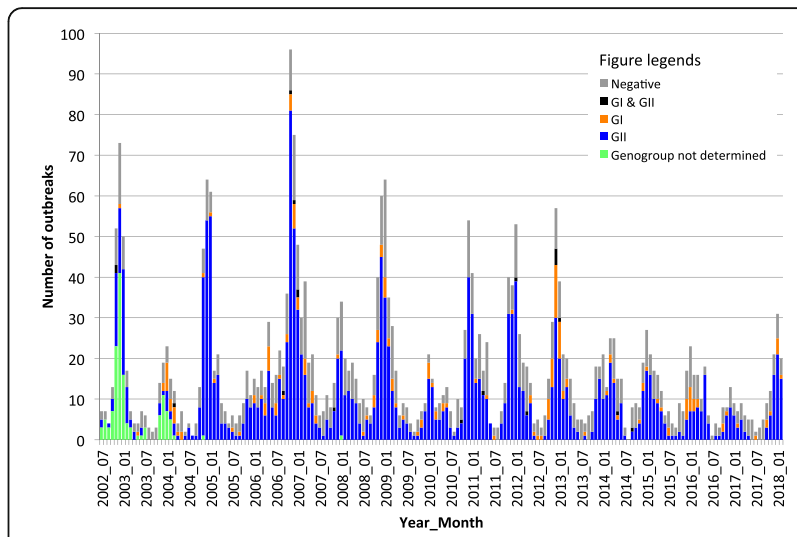

Fig. 1 Monthly distribution of norovirus-positive outbreaks in Alberta by genogroup. Data from years July 2002 to June 2012 were reported previously by Pang et al. [31] and Hasing et al. [8]. The data from this study corresponds to the period July 2012 to February 2018

Compared to historical data, the annual numbers of NoV outbreaks between July 2012 to June 2017 were lower than those observed in the previous 5 years (July 2007 to June 2012 vs. July 2012 to June 2017, $p=0.0489$, one-tailed t-test).

Genogroup II strains were responsible for 440 out of $530(83.0 \%)$ of laboratory confirmed NoV outbreaks (Table 1), while genogroup I and mixed genogroup I and genogroup II strains were responsible for 83 (15.7\%) and 7 (1.3\%) NoV outbreaks, respectively. Twenty ORF2-based genotypes were identified throughout the study period: GI.1, GI.2, GI.3, GI.4, GI.5, GI.6, GI.7, GI.9, GII.1, GII.2, GII.3, GII.4, GII.5, GII.6, GII.7, GII.8, GII.13, GII.14, GII.16 and GII.17 (Table 2). Overall, GII.4 was the most common genotype and was responsible for at least $319(60.2 \%)$ out of 530 NoV-positive outbreaks. Strains carrying an ORF2 of variant Sydney were accountable for the majority of GII.4 outbreaks (297/319, 93.1\%). GII.4 variants Den Haag and New Orleans, which caused pandemics in 2006 and 2009, respectively, disappeared after June 2014. Genotypes GI.6 and GI.7 had an increased presence during July 2012 to June 2013 whereas GI.3 was the predominant GI strain from July 2015 to June 2016. Four novel NoV strains emerged during the last three annual periods of study (Fig. 2): 1) GII.17; 2) GII.P16/GII.4 Sydney, a recombinant strain carrying a polymerase gene of GII.16 and a GII.4 Sydney capsid gene; 3) GII.P16/GII.2, a recombinant strain carrying a GII.16 polymerase gene and a GII.2 capsid gene and 4) GII.P4 New Orleans/GII.4 Sydney, a recombinant strain with a GII.4 New Orleans polymerase gene and a GII.4 Sydney capsid gene.

\section{NoV GII.17 outbreaks in Alberta}

The first GII.17 outbreak in Alberta occurred in September 2014. Phylogenetic analysis of complete capsid 
Table 1 Distribution of norovirus outbreaks by genogroups

\begin{tabular}{|c|c|c|c|c|c|c|c|}
\hline Genogroup & $\begin{array}{l}\text { July 2012-June } \\
2013\end{array}$ & $\begin{array}{l}\text { July 2013-June } \\
2014\end{array}$ & $\begin{array}{l}\text { July 2014-June } \\
2015\end{array}$ & $\begin{array}{l}\text { July 2015-June } \\
2016\end{array}$ & $\begin{array}{l}\text { July 2016-June } \\
2017\end{array}$ & $\begin{array}{l}\text { July 2017- Feb } \\
2018\end{array}$ & Total \\
\hline$\overline{\mathrm{Gl}}$ & 40 & 6 & 6 & 18 & 4 & 9 & 83 \\
\hline Gll & 103 & 96 & 85 & 60 & 35 & 61 & 440 \\
\hline Mixed $\mathrm{Gl}$ and $\mathrm{Gll}$ & 5 & 1 & 1 & 0 & 0 & 0 & 7 \\
\hline TOTAL & 148 & 103 & 92 & 78 & 39 & 70 & 530 \\
\hline Gl sequenced (\%) & $34(75.6)$ & $5(71.4)$ & $5(71.4)$ & $17(94.4)$ & $1(25)$ & $8(88.9)$ & $70(77.8)$ \\
\hline Gll sequenced (\%) & $100(92.6)$ & $87(89.7)$ & 77 (89.5) & $55(91.7)$ & $33(94.3)$ & $60(98.4)$ & $412(92.2)$ \\
\hline
\end{tabular}

sequences identified all GII.17 strains as part of the Kawasaki cluster (Additional file 1: Figure S1). Representatives of two sub-clusters of GII.17 Kawasaki previously defined by Chan et al. [23] were identified: strains from 2014 grouped into the Kawasaki323-like sub-cluster (0.7-1\% nucleotide and 0.7-0.9\% amino acid p-distances with AB983218), and strains from 2015 and 2016 grouped into the Kawasaki308-like sub-cluster (0.4-0.9\% nucleotide and $0.2-0.6 \%$ amino acid p-distances with LC037415). GII.17 was responsible for 4.3, 11.5 and 25.6 (\%) of all NoV outbreaks during the periods of July 2014-June 2015, July 2015-June 2016 and July 2016June 2017, respectively. No GII.17 outbreaks were observed after April 2017.

\section{Novel GII.P16/GII.4 Sydney recombinant}

Several GII.4 strains causing outbreaks in early 2016 could not be classified at the variant level by the genotyping tool based solely on region $\mathrm{C}$ sequences. BLAST analysis with additional sequence data from the 3'end of ORF1 ( 740 nt) revealed a high nucleotide identity (99\%) with Kawasaki194-like sequences (GenBank accession number LC175468), previously characterized as GII.P16/GII.4 Sydney recombinant strains [24]. Phylogenetic trees of partial ORF1 and partial ORF2 sequences of this recombinant are shown on Fig. 3. The complete capsid sequences of four GII.P16/GII.4 Sydney recombinants collected between 2016 and 2018, AlbertaEI84/2016, AlbertaEI277/2016, AlbertaEI487/2017 and AlbertaEI231/ 2018, had 3.3-3.9\% nucleotide and $1.5-2.2 \%$ amino acid p-distances with the GII.Pe/GII.4 Sydney 2012 reference strain NSW0514/AU/2012 (accession number JX459908) (Additional file 2: Figure S2). Compared with GII.Pe/GII.4 Sydney, the GII.P16/GII.4 Sydney strains did not present distinctive mutations at the P2 domain, however, all GII.P16/GII.4 Sydney sequences in our data set shared two isoleucines at residues 119 and 145 , both located at the $S$ domain (Additional file 3: Figure S3). The first GII.P16/GII.4 Sydney outbreak in Alberta occurred in February 2016. Overall, the GII.P16/GII.4 Sydney recombinant was responsible for $21(27.0 \%)$ of $78 \mathrm{NoV}$ outbreaks from July 2015-June 2016, 3 (7.7\%) out of $39 \mathrm{NoV}$ outbreaks from July 2016-June 2017 and 44 (62.9\%) out of $70 \mathrm{NoV}$ outbreaks from July 2017-February 2018.

\section{Saliva binding profile of the novel GII.P16/GII.4 Sydney recombinant strain}

In order to investigate the HBGA binding properties of the novel GII.P16/GII.4 Sydney recombinant, we performed binding assays using p-particles of two representative strains, $\mathrm{AB}-2016-26$ and $\mathrm{AB}-2016-190$ and saliva of individuals with known HBGA phenotypes. Both strains demonstrated ability to bind saliva of $\mathrm{A}, \mathrm{B}$ and $\mathrm{O}$ secretors and did not bind saliva of non-secretors (Additional file 4: Figure S4). AB-2016-190 showed stronger binding to secretors than AB-2016-26, but both strains showed no binding to non-secretors, similar to the binding pattern of Syd9-2B, a GII.Pe/GII.4 Sydney strain.

\section{Novel GII.P16/GII.2 recombinant}

Based on retrospective ORF1-genotyping analysis of GII.2 outbreaks back to November 2011, we identified that the first GII.P16/GII.2 outbreak in Alberta occurred in August 2016. In BLAST analysis, the GII.P16/GII.2 outbreak strain shared highest identity (99\%) with Volvic-E15317, a strain collected in France in late 2016 (Fig. 4). GII.P16/GII.2 was the predominant NoV strain between July 2016-June 2017 (13 of 39 NoV outbreaks, $33.3 \%)$.

\section{GII.P4 New Orleans/GIl.4 Sydney recombinant}

The recombinant strain GII.P4 New Orleans/GII.4 Sydney was identified during the period July 2016-June 2017 (Fig. 3). The strain was responsible for four out of five GII.4 outbreaks from that period and was not observed after June 2017.

\section{NoV outbreak settings}

Between July 2012 and February 2018, the majority of outbreaks occurred in community long-term care, hospital long-term care, supportive living, and group homes facilities (63/83, 75.9\% of all NoV GI and 346/440, 78.6\% of all NoV GII outbreaks, respectively) and hospital 
Table 2 Distribution of Norovirus strains by ORF1 and ORF2 genotype combinations

\begin{tabular}{|c|c|c|c|c|c|c|c|}
\hline ORF1/ORF2 genotypes & $\begin{array}{l}\text { July } 2012- \\
\text { June } 2013\end{array}$ & $\begin{array}{l}\text { July } 2013- \\
\text { June } 2014\end{array}$ & $\begin{array}{l}\text { July 2014- } \\
\text { June } 2015\end{array}$ & $\begin{array}{l}\text { July 2015- } \\
\text { June } 2016\end{array}$ & $\begin{array}{l}\text { July 2016- } \\
\text { June } 2017\end{array}$ & $\begin{array}{l}\text { July 2017- } \\
\text { Feb } 2018\end{array}$ & Total \\
\hline \multicolumn{8}{|l|}{ Gl sequenced } \\
\hline Pol NT/Gl.1 & & & 1 & & & & 1 \\
\hline GI.P1/GI.1 & & & & & & 3 & 3 \\
\hline Pol NT/GI.2 & & 2 & & 1 & & & 3 \\
\hline GI.2/GI.P2 & & & & & 1 & & 1 \\
\hline Pol NT/GI.3 & & 1 & 1 & 15 & & & 17 \\
\hline GI.P3/GI.3 & & & & & & 4 & 4 \\
\hline Pol NT/GI.4 & & 1 & & & & & 1 \\
\hline Pol NT/GI.5 & & & 2 & & & & 2 \\
\hline Pol NT/GI.6 & 20 & & 1 & & & & 21 \\
\hline $\mathrm{Gl} . \mathrm{Pb} / \mathrm{Gl} .6$ & & & & & & 1 & 1 \\
\hline Pol NT/GI.7 & 14 & & & 1 & & & 15 \\
\hline Pol NT/GI.9 & & 1 & & & & & 1 \\
\hline \multicolumn{8}{|l|}{ Gll sequenced } \\
\hline Pol NT/GIl.1 & & & & 1 & & & 1 \\
\hline Gll.P16/GIl.2 & & & & & 13 & & 13 \\
\hline GII.P2/GII.2 & 1 & & & 1 & & & 2 \\
\hline Gll.Pe/GII.2 & & & & 1 & & & 1 \\
\hline Pol NT/GII.3 & & & & 1 & & & 1 \\
\hline GII.P12/GIl.3 & & & & & 1 & 3 & 4 \\
\hline Pol NT/GII.4 Den Haag 2006b & 4 & 1 & & & & & 5 \\
\hline Pol NT/GIl.4 New Orleans 2009 & 5 & 7 & & & & & 12 \\
\hline GII.P4 New Orleans 2009/GIl.4 New Orleans 2009 & 1 & 4 & & & & & 5 \\
\hline Pol NT/GIl.4 Sydney 2012 & 86 & 54 & 51 & 9 & & 4 & 204 \\
\hline GIl.P16/GIl.4 Sydney 2012 & & & & 21 & 3 & 44 & 68 \\
\hline GII.P4 New Orleans 2009/GIl.4 Sydney 2012 & & & & & 4 & & 4 \\
\hline Gll.Pe/Gll.4 Sydney 2012 & & 2 & 9 & 3 & 2 & 5 & 21 \\
\hline Pol NT/GIl.5 & & 7 & 1 & & & & 8 \\
\hline Pol NT/Gll.6 & 2 & 7 & 5 & 5 & & & 19 \\
\hline GII.P7/GII.6 & & & & & & 3 & 3 \\
\hline Pol NT/GIl.7 & 1 & & 1 & 2 & & & 4 \\
\hline Gll.P7/GII.7 & & & & & & 1 & 1 \\
\hline Pol NT/GII.8 & & 1 & 1 & 1 & & & 3 \\
\hline Pol NT/GII.13 & & 4 & 4 & & & & 8 \\
\hline Pol NT/GII.14 & & & 1 & & & & 1 \\
\hline Pol NT/GII.16 & & & & 1 & & & 1 \\
\hline Pol NT/GII.17 & & & 4 & 9 & 6 & & 19 \\
\hline GII.P17/GII.17 & & & & & 4 & & 4 \\
\hline
\end{tabular}

Pol NT = polymerase not typed

acute care $(5 / 83,6 \%$ of GI and 53/440,12\% of GII outbreaks) (Additional file 5: Figure S5). A larger proportion of GI outbreaks occurred in non-health care settings compared to GII $(15 / 83,18.1 \%$ vs. $41 / 440,9.3 \%$, $p=0.0180$, Chi-square test).

\section{Discussion}

During July 2012 to February 2018 the NoV outbreak activity in Alberta presented important changes in terms of overall disease burden and $\mathrm{NoV}$ genotype distribution as compared to historical data. Between 


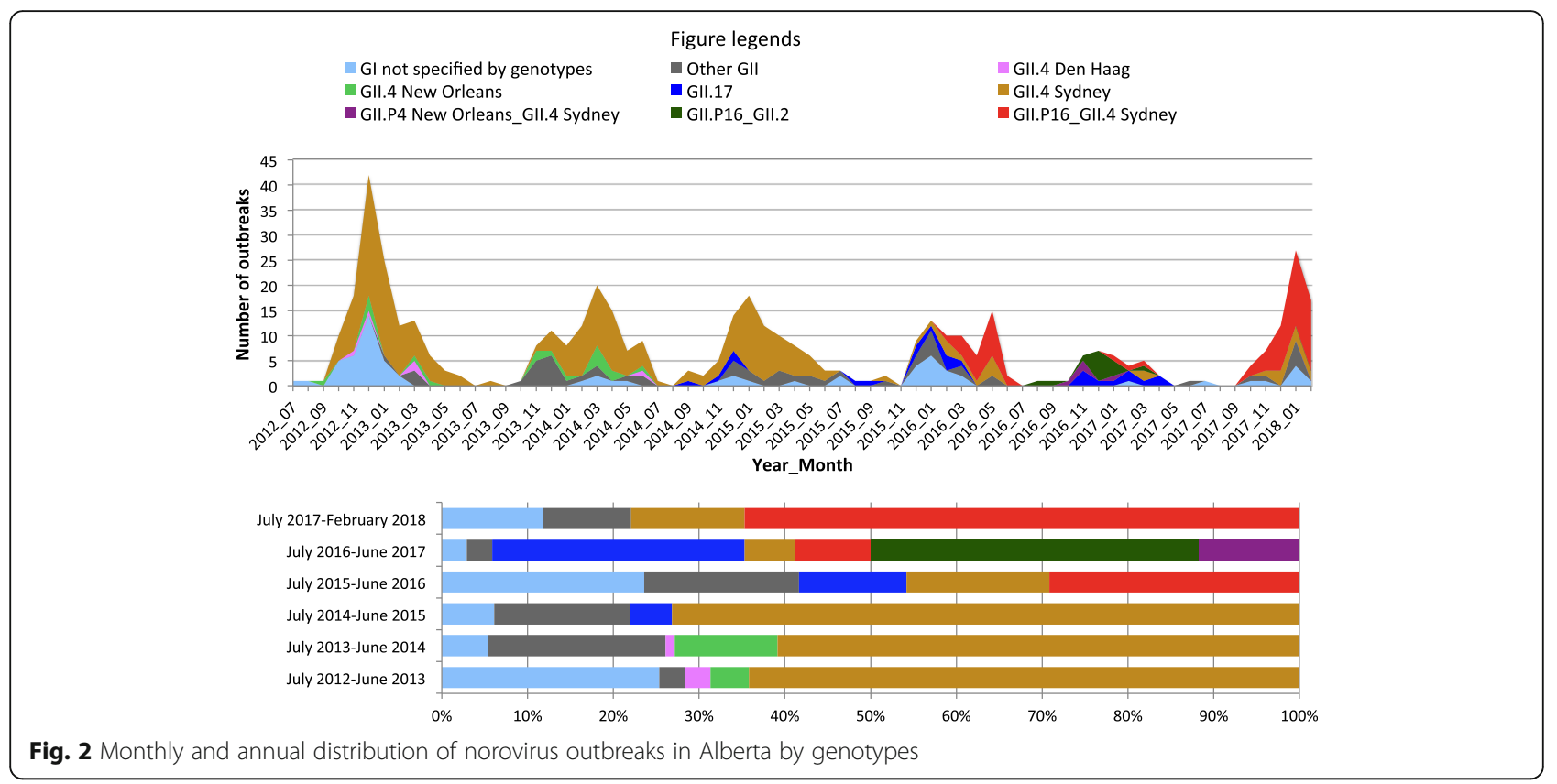

July 2012 and June 2017, the annual numbers of NoV outbreaks had a gradual decline. Genotype GII.4 outbreaks, which have previously represented over 50\% of all NoV outbreaks, decreased to $23 \%$ in July $2016-$ June 2017. The steep decline in NoV outbreaks was followed by rebound in July 2017-February 2018, when GII.4 outbreaks were accountable for $76 \%$ of all NoV outbreaks.
GII.4 Sydney remained as the single predominant strain responsible for a large majority $(>50 \%)$ of all $\mathrm{NoV}$ outbreaks from July 2012 to June 2015. In contrast, the following 2 years, July 2015 to June 2017, had an atypical high proportion of non-GII.4 outbreaks. Moreover, four novel NoV strains emerged in the province: GII.17 Kawasaki, GII.P16/GII.4 Sydney, GII.P16/GII.2, and GII.P4 New Orleans/GII.4 Sydney.

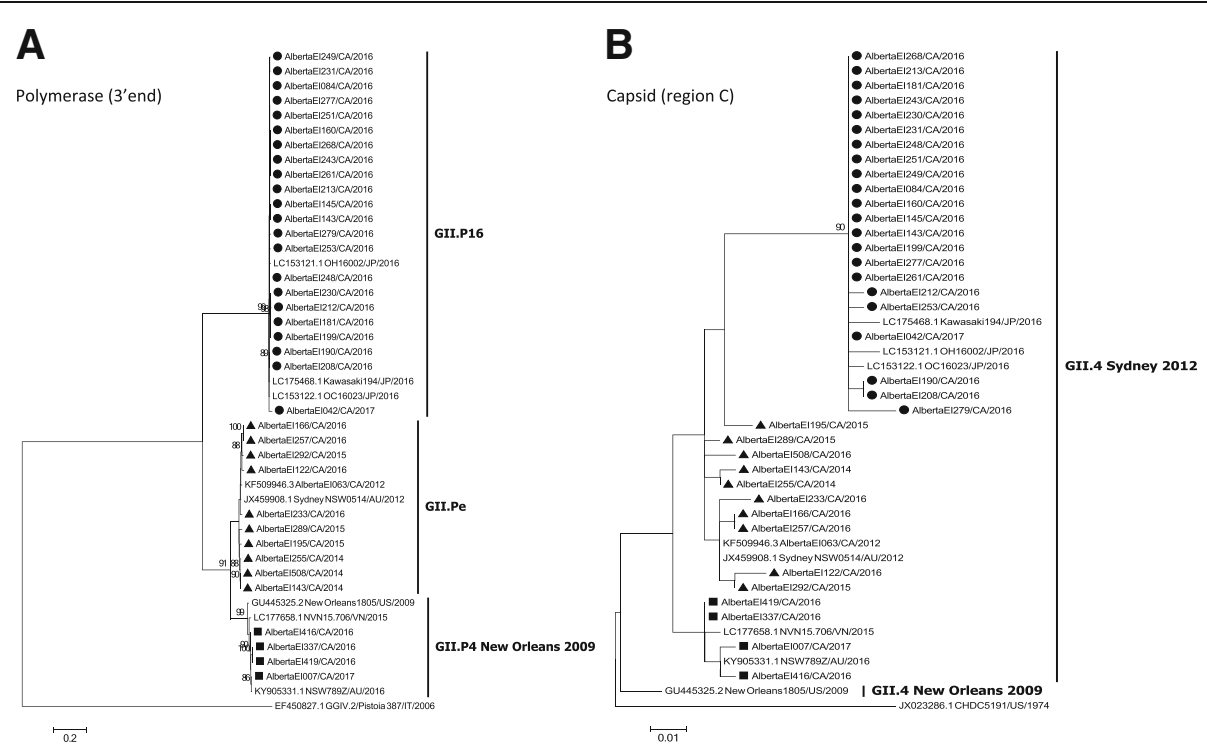

Fig. 3 Maximum likelihood phylogenies of recombinant Gll.4 strains that emerged in Alberta. Three different Gll.4 Sydney recombinant strains were identified based on sequences of (a) the 3'end of ORF1 and (b) region C of the capsid gene. The novel GII.P16/GIl.4 Sydney strains are shown with solid circles; GII.P4 New Orleans/GIl.4 Sydney and GII.Pe/GIl.4 Sydney strains are shown with solid squares and solid triangles, respectively. Trees A and B were constructed using the Kimura-2 parameter substitution model assuming gamma-distributed rates of evolution among sites with (tree A) and without (tree B) invariant sites. Branch significance was estimated based on 1000 bootstrap replicates 


\section{A}

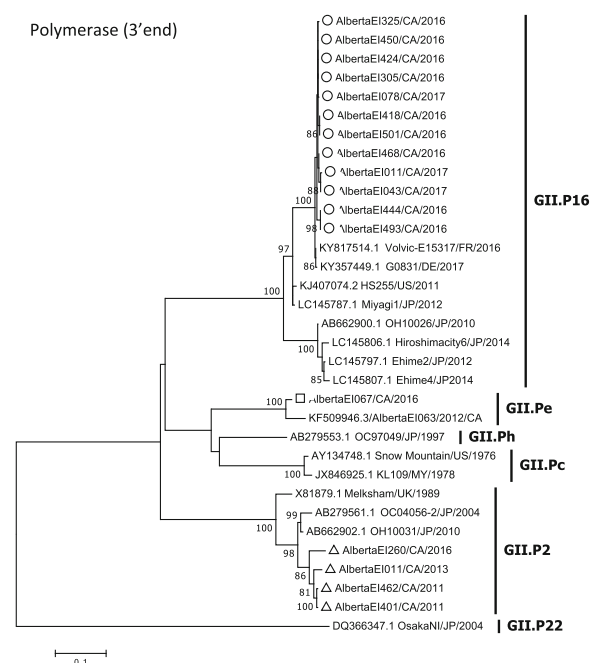

B

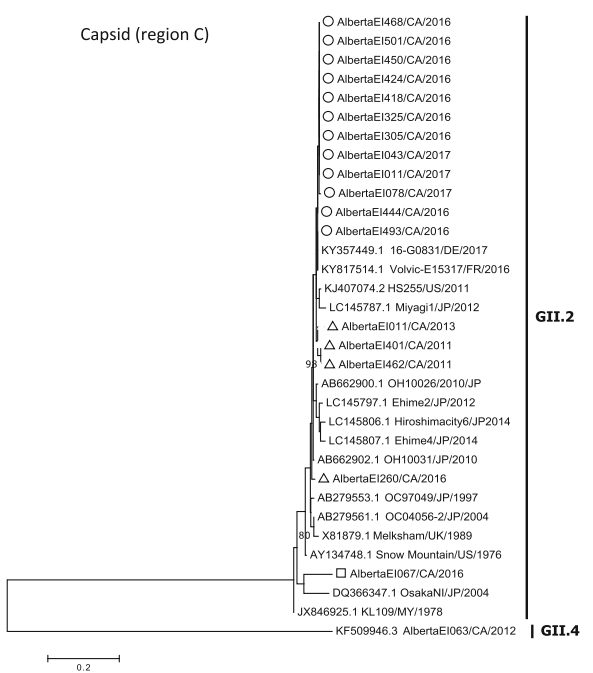

Fig. 4 Maximum likelihood phylogenies of norovirus GIl.2 strains. Gll.2 sequences at (a) the 3'end of ORF1 and (b) region C of the capsid gene were analyzed. The novel GII.P16/GIl.2 recombinant sequences are shown with open circles; GII.Pe.GIl.2 and GIl.P2/GIl.2 strains are shown, respectively, with open squares and open triangles. All trees were constructed using the Kimura-2 parameter substitution model assuming gamma-distributed rates of evolution among sites with (tree A) and without (tree B) invariant sites. Branch significance was estimated based on 1000 bootstrap replicates

The GII.17 Kawasaki strain was first reported in Asia in September 2014 where it quickly became the predominant genotype during the 2014-2015-winter season raising concern of a global pandemic [10]. Since its emergence, GII.17 Kawasaki has undergone diversification into at least three sub-clusters [10]. In Alberta, we identified two sub-clusters: the Kawasaki 308-like sub-cluster and the Kawasaki 323-like sub-cluster. Although both clusters started to circulate in the province at similar timeframes as reported in Asia [10], the GII.17 Kawasaki had limited prevalence in Alberta, as also observed in other regions outside Asia, including Europe and the United States [10]. The factors limiting the transmission of GII.17 Kawasaki outside Asia are largely unknown. The GII.17 Kawasaki strains display a broad saliva HBGA-binding profile comparable to pandemic GII.4 strains [25], which emphasizes the potential of GII.17 as a pandemic strain.

The novel GII.P16/GII.4 Sydney recombinant was the most common strain among NoV outbreaks from July 2015-June 2016. Although the prevalence of this recombinant decreased temporarily in July 2016-June 2017, its circulation increased sharply in the more recent period, July 2017 and February 2018, when GII.P16/GII.4 Sydney was again responsible for a large majority of outbreaks. The GII.P16/GII.4 Sydney recombinant also circulated in the United States and Germany during the 2015-2016 winter season $[18,26]$. Our analysis of capsid sequences from this recombinant collected at three different time points (2016, 2017 and 2018) demonstrated no unique amino acid substitutions compared to GII.Pe/ GII.4 Sydney, its ORF2 parental strain. Furthermore, we observed that GII.P16/GII.4 Sydney can bind to saliva of secretor HBGA phenotypes but not to that of non-secretors, similarly as GII.Pe/GII.4 Sydney strains. Our findings agree with a recent phylogenetic study [27] and suggest that amino acid substitutions outside ORF2, rather than antigenic change at the capsid gene, conferred an evolutionary advantage to GII.P16/GII.4 Sydney over its parental strain GII.Pe/GII.4 Sydney.

The GII.P4 New Orleans/GII.4 Sydney recombinant had low circulation between July 2016 and June 2017 and was no longer observed in the subsequent period. We hypothesize that the transmission of this recombinant in Alberta might have been restricted by its antigenic resemblance to two highly circulated strains, GII.4 New Orleans and GII.4 Sydney. GII.4 has been the predominant genotype worldwide among humans since the 1990s but had very limited circulation in the 1970s and 1980s $[28,29]$. It is possible that the decline in GII.4 NoV outbreaks observed between July 2015 and June 2017 is a consequence of herd immunity accumulated in the population after two decades of high prevalence of various GII.4 variants and exhaustion of mutational sites in GII.4 Sydney. The GII.P16/GII.4 Sydney recombinant might have partially circumvented such factors by acquiring the ORF1 genes from the less circulating, and presumably more fit, GII.P16. Further surveillance data is still required to understand the turnover of NoV genotypes in humans. 
The last recombinant that emerged in Alberta in the time period of the present study is GII.P16/GII.2. Although GII.P16/GII.2 outbreaks have been reported previously in Asia in 2009, phylogenetic time-scale analyses performed by others suggest that the polymerase of the recent GII.P16/GII.2 strain is rather closely related to that of GII.P16/GII.4 Sydney and carries amino acid substitutions that could have conferred the novel GII.P16/ GII.2 an evolutionary advantage [30]. GII.P16/GII.2 strains have also been reported in Germany, France, Japan, China and the United States since mid-2016 [18, 30]. Notably, GII.P16/GII.2 was the predominant strain in the province briefly in July 2016-June 2017, but was replaced thereafter by GII.P16/GII.4 Sydney.

A limitation of our study is the limited collection of ORF1 data for the period between July 2012 and June 2015, which could have lead us to miss novel or unusual recombinants. However, we believe the likelihood of missing important strains is low since $69 \%$ of the norovirus GII outbreaks in Alberta within those years were caused by a single ORF2 genotype, GII.4 Sydney. Reports from North America [18] and diverse countries around different continents [20] suggest that GII.Pe/GII.4 Sydney was the major strain in circulation worldwide during that time frame and thus, we believe that most, if not all of GII.4 Sydney from that period, carried a GII.Pe polymerase.

\section{Conclusions}

In summary, we provided 6 years of systematic molecular surveillance data of norovirus outbreaks in Alberta and identified a GII.4 recombinant, GII.P16/GII.4 Sydney, as the most prevalent strain causing outbreaks in the province in early 2018. An important shift and increase in genotype diversity was noticed in recent years, which should be considered for vaccine development. Ongoing surveillance of the molecular epidemiology of NoV using a dual ORF1/ORF2 genotyping scheme is indispensable to better identify the disease burden of new emerging strains and understand their evolutionary pathways.

\section{Additional files}

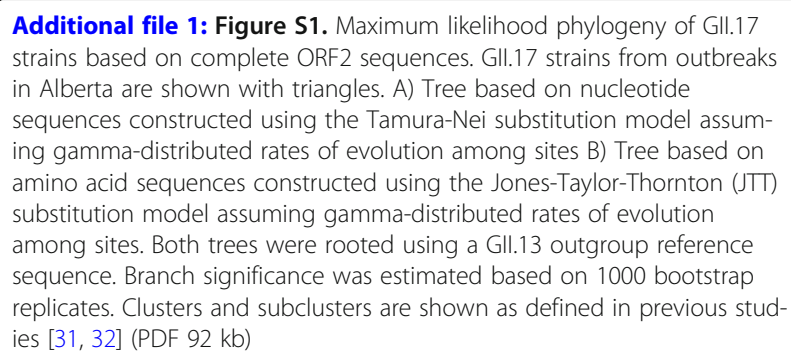

Additional file 2: Figure S2. Maximum likelihood phylogeny of complete GII.P16/GIl.4 Sydney capsid sequences. The maximum-likelihood tree was constructed using the Tamura-Nei substitution model, assuming gammadistributed rates of evolution among sites. The analysis included strains representative of different countries and different years. (PDF $14 \mathrm{~kb}$ )

Additional file 3: Figure S3. Description of data: Analysis of variable sites in capsid sequences of GII.P16/Gll.4 Sydney recombinants compared to GII.Pe/GIl.4 Sydney strains. Tables depict all variable amino acid residues identified in the alignment used to construct the tree shown in Additional file 2. Evolving amino acid positions previously reported by Lindesmith et al. [33] to play a role in the evasion of antibody immune responses are shown in red. (PDF $80 \mathrm{~kb}$ )

Additional file 4: Figure S4. Saliva-binding of NoV P-domain proteins. The P-domain proteins of (A) AB-2016-26 (GIl.P16/Gll.4 Sydney), (B) AB2016-190 (GII.P16/GII.4 Sydney) and (C) Syd9-2B (GII.Pe/GIl.4 Sydney) were tested in their ability to bind saliva from individuals with different HBGAs profiles. (PDF $255 \mathrm{~kb}$ )

Additional file 5: Figure S5. Norovirus outbreak settings in Alberta by genogroup. Outbreaks from mixed Gl and Gll strains $(n=7)$ were excluded from the analysis. (PDF $23 \mathrm{~kb}$ )

\section{Abbreviations}

GST: Glutathione S-transferase; HBGAs: Histo-blood group antigens; HRP: Horseradish peroxidase; NoV: Norovirus; ORF: Open reading frame; ProvLab: Provincial Laboratory for Public Health; RT: Reverse transcription

\section{Acknowledgements}

We thank Andy Wang, Jay Gamma, Joanne Cho and Oliver Jin for their assistance with norovirus sequencing. We also thank the Provincial Laboratory for Public Health staff, especially the virology and molecular departments performing testing for norovirus and the collaborative teams participating in outbreak investigations in Alberta, Northwest Territories, Yukon, and Nunavut, and the members of the Public Health (ProvLab) Outbreak Investigation Committee (OINC).

\section{Funding}

This work was supported by the Provincial Laboratory for Public Health, Alberta, Canada, and Alberta Health, Canada. The Provincial Laboratory for Public Health (ProvLab) runs the norovirus outbreak molecular surveillance program in Alberta. ProvLab scientists participated in the study design, collection, analysis and interpretation of data as well as writing the manuscript. Alberta Health had no role in the study design, data collection and analysis or manuscript preparation.

\section{Availability of data and materials}

The norovirus complete capsid sequences obtained during the current study are available in GenBank under accession numbers MH922873 through MH922876.

\section{Authors' contributions}

$\mathrm{MH}$ performed phylogenetic and statistical analyses and drafted the manuscript.

BL developed and maintained the study database and contributed with data analysis. MX and XJ contributed with norovirus P-particle expression and saliva binding studies. YQ, KP and AW conducted norovirus genotyping. GT provided critical input to the discussion and result interpretation. XP developed the concept of the study and coordinated data collection. All authors reviewed and contributed to the manuscript draft and approved the final version.

\section{Ethics approval and consent to participate}

This study was approved by the University of Alberta Biomedical Health Research Ethics Board. The board waived the need of participant consent because samples were anonymized (study ID Pro00037093).

\section{Consent for publication}

Not applicable. 


\section{Competing interests}

The authors declare that they have no competing interests.

\section{Publisher's Note}

Springer Nature remains neutral with regard to jurisdictional claims in published maps and institutional affiliations.

\section{Author details}

'Department of Laboratory Medicine and Pathology, University of Alberta Edmonton, AB, Canada. ${ }^{2}$ Department of Paediatrics, University of Alberta, Edmonton, AB, Canada. ${ }^{3}$ Division of Infectious Diseases, Cincinnati Children's Hospital Medical Centre, Cincinnati, OH, USA. ${ }^{4}$ Department of Medical Microbiology and Immunology, University of Alberta, Edmonton, AB, Canada. ${ }^{5}$ Provincial Laboratory for Public Health (Microbiology), University of Alberta Hospital, WMC 2B4.58, 8440-112 Street, Edmonton, Alberta T6G 2J2, Canada.

Received: 24 September 2018 Accepted: 7 February 2019

Published online: 19 February 2019

\section{References}

1. Pires SM, Fischer-Walker CL, Lanata CF, Devleesschauwer B, Hall AJ, Kirk MD, Duarte AS, Black RE, Angulo FJ. Aetiology-specific estimates of the global and regional incidence and mortality of Diarrhoeal diseases commonly transmitted through food. PLoS One. 2015;12:e0142927.

2. Bartsch SM, Lopman BA, Ozawa S, Hall AJ, Lee BY. Global economic burden of norovirus gastroenteritis. PLoS One. 2016;4:e0151219.

3. Ahmed SM, Hall AJ, Robinson AE, Verhoef L, Premkumar P, Parashar UD, Koopmans M, Lopman BA. Global prevalence of norovirus in cases of gastroenteritis: a systematic review and meta-analysis. Lancet Infect Dis. 2014;8:725-30

4. Siebenga JJ, Vennema H, Renckens B, de Bruin E, van der Veer B, Siezen RJ, Koopmans M. Epochal evolution of GGll.4 norovirus capsid proteins from 1995 to 2006. J Virol. 2007:18:9932-41.

5. Eden JS, Tanaka MM, Boni MF, Rawlinson WD, White PA. Recombination within the pandemic norovirus Gll.4 lineage. J Virol. 2013;87:6270-82.

6. Vinje J. Advances in laboratory methods for detection and typing of norovirus. J Clin Microbiol. 2015;2:373-81.

7. van Beek J, Ambert-Balay K, Botteldoorn N, Eden JS, Fonager J, Hewitt J, Iritani N, Kroneman A, Vennema H, Vinje J, et al. Indications for worldwide increased norovirus activity associated with emergence of a new variant of genotype II.4, late 2012. Euro Surveill. 2013;18:8-9.

8. Hasing ME, Lee BE, Preiksaitis JK, Tellier R, Honish L, Senthilselvan A, Pang $\mathrm{XL}$. Emergence of a new norovirus Gll.4 variant and changes in the historical biennial pattern of norovirus outbreak activity in Alberta, Canada, from 2008 to 2013. J Clin Microbiol. 2013;7:2204-11.

9. Siebenga JJ, Vennema H, Zheng DP, Vinje J, Lee BE, Pang XL, Ho EC, Lim W, Choudekar A, Broor $\mathrm{S}$, et al. Norovirus illness is a global problem: emergence and spread of norovirus Gll.4 variants, 2001-2007. J Infect Dis. 2009;5:802-12

10. Chan MCW, Hu Y, Chen H, Podkolzin AT, Zaytseva EV, Komano J, Sakon N, Poovorawan Y, Vongpunsawad S, Thanusuwannasak T, et al. Global spread of norovirus Gll.17 Kawasaki 308, 2014-2016. Emerg Infect Dis. 2017:8:1359-4.

11. Debbink K, Lindesmith LC, Donaldson EF, Costantini V, Beltramello M, Corti D, Swanstrom J, Lanzavecchia A, Vinje J, Baric RS. Emergence of new pandemic Gll.4 Sydney norovirus strain correlates with escape from herd immunity. J Infect Dis. 2013:11:1877-87.

12. Parra Gl, Squires RB, Karangwa CK, Johnson JA, Lepore CJ, Sosnovtsev SV, Green KY. Static and evolving norovirus genotypes: implications for epidemiology and immunity. PLoS Pathog. 2017;1:e1006136.

13. Yang $Y$, Xia M, Tan $M$, Huang $P$, Zhong W, Pang XL, Lee BE, Meller J, Wang $T$, Jiang $X$. Genetic and phenotypic characterization of Gll-4 noroviruses that circulated during 1987 to 2008. J Virol. 2010;18:9595-607.

14. Pang XL, Preiksaitis JK, Lee B. Multiplex real time RT-PCR for the detection and quantitation of norovirus genogroups I and II in patients with acute gastroenteritis. J Clin Virol. 2005;2:168-71.

15. Kojima S, Kageyama T, Fukushi S, Hoshino FB, Shinohara M, Uchida K, Natori K, Takeda N, Katayama K. Genogroup-specific PCR primers for detection of Norwalk-like viruses. J Virol Methods. 2002;1-2:107-14.
16. Iritani N, Kaida A, Kubo H, Abe N, Murakami T, Vennema H, Koopmans M, Takeda N, Ogura H, Seto Y. Epidemic of genotype Gll.2 noroviruses during spring 2004 in Osaka City, Japan. J Clin Microbiol. 2008;7:2406-9.

17. Kageyama T, Kojima S, Shinohara M, Uchida K, Fukushi S, Hoshino FB, Takeda N, Katayama K. Broadly reactive and highly sensitive assay for Norwalk-like viruses based on real-time quantitative reverse transcriptionPCR. J Clin Microbiol. 2003:4:1548-57.

18. Cannon JL, Barclay L, Collins NR, Wikswo ME, Castro CJ, Magana LC, Gregoricus N, Marine RL, Chhabra P, Vinje J. Genetic and Epidemiologic Trends of Norovirus Outbreaks in the United States from 2013 to 2016 Demonstrated Emergence of Novel Gll.4 Recombinant Viruses. J Clin Microbiol. 2017;7:2208-21.

19. Kroneman A, Vennema $H$, Deforche $K$, v d Avoort $H$, Penaranda S, Oberste MS, Vinje J, Koopmans M An automated genotyping tool for enteroviruses and noroviruses. J Clin Virol 2011;2:121-125.

20. van Beek J, de Graaf M, Al-Hello H, Allen DJ, Ambert-Balay K, Botteldoorn N, Brytting M, Buesa J, Cabrerizo M, Chan M, et al. Molecular surveillance of norovirus, 2005-16: an epidemiological analysis of data collected from the NoroNet network. Lancet Infect Dis. 2018;18:545-53.

21. Tamura K, Stecher G, Peterson D, Filipski A, Kumar S. MEGA6: molecular evolutionary genetics analysis version 6.0. Mol Biol Evol. 2013;12:2725-9.

22. Tan $M$, Jiang $X$. The $p$ domain of norovirus capsid protein forms a subviral particle that binds to histo-blood group antigen receptors. J Virol. 2005;22: 14017-30.

23. Chan MC, Lee N, Hung TN, Kwok K, Cheung K, Tin EK, Lai RW, Nelson EA, Leung TF, Chan PK. Rapid emergence and predominance of a broadly recognizing and fast-evolving norovirus Gll.17 variant in late 2014. Nat Commun. 2015;6:10061.

24. Matsushima Y, Shimizu T, Ishikawa M, Komane A, Okabe N, Ryo A, Kimura H, Katayama K, Shimizu H. Complete Genome Sequence of a Recombinant GII. P16-Gll.4 Norovirus Detected in Kawasaki City, Japan, in 2016. Genome Announc. 2016;4:5

25. Zhang XF, Huang Q, Long Y, Jiang X, Zhang T, Tan M, Zhang QL, Huang ZY, $\mathrm{Li} Y H$, Ding YQ et al. An outbreak caused by Gll.17 norovirus with a wide spectrum of HBGA-associated susceptibility. Sci Rep. 2015;17687.

26. Niendorf S, Jacobsen S, Faber M, Eis-Hubinger AM, Hofmann J, Zimmermann O, Hohne M, Bock CT. Steep rise in norovirus cases and emergence of a new recombinant strain GII.P16-GIl.2, Germany, winter 2016. Euro Surveill. 2017:22:4

27. Ruis C, Roy S, Brown JR, Allen DJ, Goldstein RA, Breuer J. The emerging GIl. P16-Gll.4 Sydney 2012 norovirus lineage is circulating worldwide, arose by late-2014 and contains polymerase changes that may increase virus transmission. PLoS One. 2017;6:e0179572.

28. Bok K, Abente EJ, Realpe-Quintero M, Mitra T, Sosnovtsev SV, Kapikian AZ, Green KY. Evolutionary dynamics of Gll.4 noroviruses over a 34-year period. Jirol. 2009:22:11890-901.

29. van Beek J, de Graaf M, Xia M, Jiang X, Vinje J, Beersma M, de Bruin E, van de Vijver D, Holwerda M, van Houten M, et al. J Gen Virol. 2016.

30. Ao Y, Cong X, Jin M, Sun X, Wei X, Wang J, Zhang Q, Song J, Yu J, Cui J, et al. Genetic analysis of reemerging GII.P16-GIl.2 noroviruses in 2016-2017 in China. J Infect Dis. 2018;1:133-43.

31. Pang XL, Preiksaitis JK, Wong S, Li V, Lee BE. Influence of novel norovirus GII. 4 variants on gastroenteritis outbreak dynamics in Alberta and the northern territories, Canada between 2000 and 2008. PLoS One. 2010;7:e11599.

32. Dang Thanh $H$, Than VT, Nguyen TH, Lim I, Kim W. Emergence of Norovirus Gll.17 Variants among Children with Acute Gastroenteritis in South Korea. PLoS One. 2016 May 5:11(5):e0154284.

33. Lindesmith LC, Brewer-Jensen PD, Mallory ML, Debbink K, Swann EW, Vinje J, Baric RS. Antigenic characterization of a novel recombinant GII.P16-Gll.4 Sydney norovirus strain with minor sequence variation leading to antibody escape. J Infect Dis. 2018;7:1145-52. 